\section{Jaundice in pregnancy}

We read with interest the case report by Stone and Girling. ${ }^{1}$ We report a case of cholangiocarcinoma diagnosed in pregnancy. A 27-year-old woman in her first pregnancy presented with progressively worsening pruritus, deepening jaundice and deranged liver function at six weeks of pregnancy. She had no nausea or abdominal pain. Her medical history was unremarkable. She had no history of inflammatory bowel disease.

On clinical examination she was deeply icteric. The liver and the gallbladder were not palpable. The early pregnancy ultrasound scan (USS) confirmed a singleton viable pregnancy. Her hepatitis viral serology, liver autoantibodies and HIV serology were negative.

The alanine transaminase level was $268 \mathrm{U} / \mathrm{L}$, total serum bilirubin $110 \mu \mathrm{mol} / \mathrm{L}$ and gamma-glutamyl transpeptidase of $1325 \mathrm{IU} / \mathrm{L}$. The carbohydrate antigen 19-9, currently the most widely used tumour marker for cholangiocarcinoma, ${ }^{2}$ was elevated (CA19-9: $1956 \mathrm{kU} / \mathrm{L}$ ).

Owing to deteriorating liver function tests (LFT), further investigations were undertaken. Abdominal and endoscopic USS were negative. The MRCP (see Figure 1) demonstrated multiple diffuse intra- and extrahepatic duct strictures and a tight hilar stricture from the common bile duct to the hepatic duct, highly suggestive of primary sclerosing cholangitis (PSC).

Subsequently endoscopic retrograde cholangiopancreatography (ERCP) was performed and microscopic examination of the brushings from the bile duct was undertaken and reported to be negative on the first occasion; however, on repeat testing atypical cells suggestive of malignancy were revealed. Computed tomography suggested a mass most likely to be cholangiocarcinoma which is a well-known complication of PSC. ${ }^{3}$

At 20 weeks the fetus was found to have severe early onset growth restriction and oligohydramnios with abnormal Doppler blood flow studies. However, the patient decided to continue the pregnancy. She was transferred to a tertiary liver unit and her diagnosis of an inoperable cholangiocarcinoma was confirmed. She delivered a live female baby by elective caesarean section at 32 weeks gestation.

Serum trypsinogen-2 is elevated in cholangiocarcinoma and is not influenced by hepatic dysfunction, biliary obstruction or pancreatic irritation. ${ }^{3}$ This was not measured in our case. Cholangiography (i.e. endoscopic retrograde cholangiography [ERC], percutaneous transhepatic cholangiography [PTC] or magnetic resonance cholangiopancreatography $[\mathrm{MRCP}])$ are the best imaging modalities to identify early cholangiocarcinoma. ${ }^{4}$ ERC or PTC allows brushing of the strictures but brush cytology has low sensitivity ${ }^{5}$ and negative biopsies and brushings do not exclude cholangiocarcinoma. ${ }^{6}$ In this case the patient had MRCP performed prior to ERCP. There is a strong association of PSC with inflammatory bowel disease and primarily ulcerative colitis. Up to $60-72 \%$ of the cases have ulcerative colitis, less commonly Crohn's disease. Patients with PSC are at increased risk (up to 15\%) for developing cholangiocarcinoma. This patient had a colonoscopy to exclude this condition.

Endoscopic US is good at identifying common bile duct stones but is less reliable above the hilum. MRCP should be

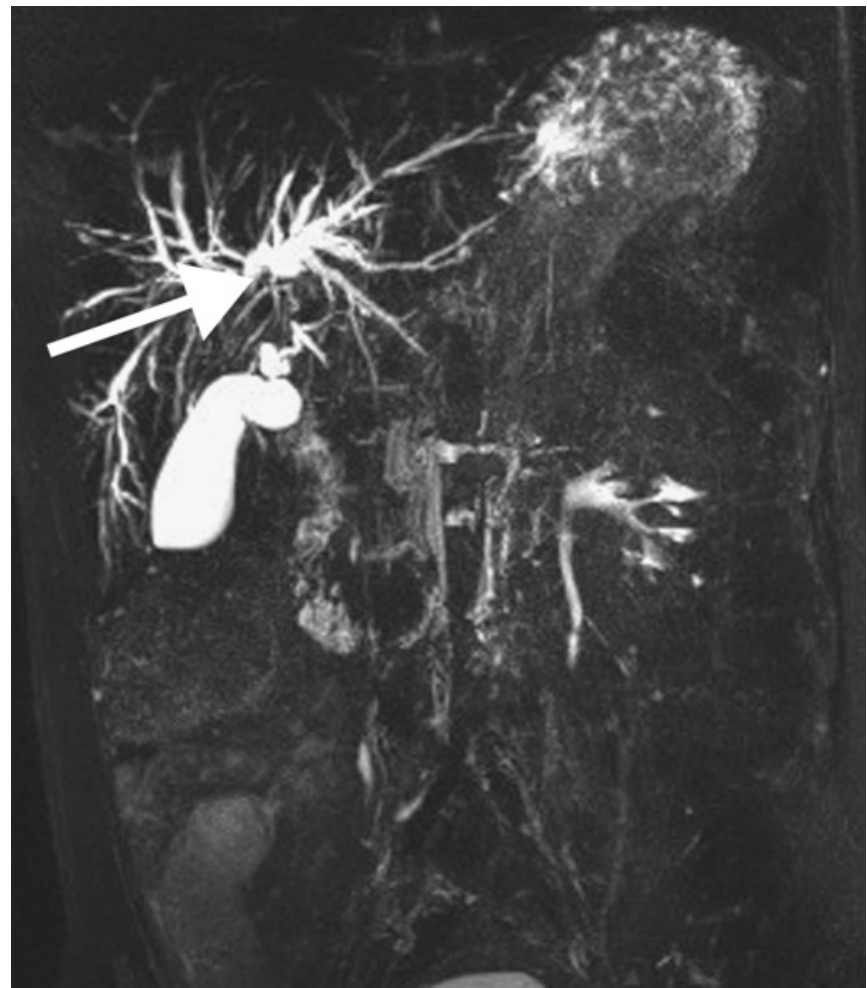

Figure 1 Magnetic resonance cholangiopancreatography (MRCP) showing Bismuth stage IV hilar stricture (arrow) and intrahepatic cholangiopathy

done in pregnancy, as it is very useful even with normal USS (which is not accurate in cholangiocarcinoma).

USS has limitations and the influence of bowel gas makes the images difficult to interpret. There is a possibility of a falsenegative result on the abdominal ultrasound.

Repeated diagnostic procedures and a high degree of clinical suspicion are sometimes needed to diagnose cholangiocarcinoma.

\section{Anita Dutta* and Terry Wong ${ }^{\dagger}$}

*Department of Obstetrics and Gynaecology, St Thomas' Hospital;

†Guy's and St Thomas' Hospitals Trust, London, UK

Correspondence to: Dr Anita Dutta

Email: anita.dutta@gstt.nhs.uk

DOI: $10.1258 /$ om.2009.090048

\section{REFERENCES}

1 Stone S, Girling JC. Deranged liver function tests in pregnancy: the importance of postnatal follow-up. Obstet Med 2009;2:32-3. doi: 10.1258/om.2008.080036

2 Duffy MJ. CA 19-9 as a marker for gastrointestinal cancers: a review. Ann Clin Biochem 1998;35:364-70

3 Hedstrom J, Haglund C, Haapiainen R, Stenman UH. Serum trypsinogen-2 and trypsin-2-alpha(1)-antitrypsin complex in malignant and benign digestive-tract diseases. Preferential elevation in patients with cholangiocarcinomas. Int J Cancer 1996;66:326-31

4 Gores GJ. Early detection and treatment of cholangiocarcinoma. Liver Transplant 2000;6:S30-4

5 Boberg KM, Schrump FE. Diagnosis and treatment of cholangiocarcinoma. Curr Gastroenterol Rep 2004;6:52-9

6 Baskin-Bey ES, Moreno Luna LE, Gores GJ. Diagnosis of cholangiocarcinoma in patients with PSC: a sight on cytology. J Hepatol 2006;45:476-9 